\title{
Novel Surface Expression of Reticulocalbin 1 on Bone Endothelial Cells and Human Prostate Cancer Cells Is Regulated by TNF- $\alpha$
}

\author{
Carlton R. Cooper, ${ }^{1 *}$ Bianca Graves, ${ }^{1}$ Freddie Pruitt, ${ }^{1}$ Hassan Chaib, ${ }^{7}$ Jill E. Lynch, ${ }^{1}$ \\ Andrew Koemeter Cox ${ }^{1}$ Linda Sequeria, ${ }^{1}$ Kenneth L. van Golen, ${ }^{1}$ Angelo Evans, ${ }^{1}$ Kirk Czymmek, ${ }^{1,2}$ \\ Rebecca S. Bullard, ${ }^{3}$ Carlton D. Donald, ${ }^{3}$ Katia Sol-Church, ${ }^{4}$ James D. Gendernalik, ${ }^{5}$ Babette Weksler, ${ }^{6}$ \\ Mary C. Farach-Carson, ${ }^{1}$ Jill A. Macoska, ${ }^{7}$ Robert A. Sikes, ${ }^{1}$ and Kenneth J. Pienta ${ }^{5,7}$ \\ ${ }^{1}$ Center for Translational Cancer Research and Department of Biological Sciences, \\ University of Delaware, Newark, Delaware \\ ${ }^{2}$ Delaware Biotechnology Institute, Department of Biological Sciences, University of Delaware, \\ Newark, Delaware \\ ${ }^{3}$ Department of Pathology and Lab Medicine, Medical University of Charleston, Charleston, South Carolina \\ ${ }^{4}$ Biomedical Research Department, Nemours Children Clinic, Wilmington, Delaware \\ ${ }^{5}$ Section of Hematology/Oncology, Departments of Internal Medicine and Urology, \\ Comprehensive Cancer Center, University of Michigan, Ann Arbor, Michigan \\ ${ }^{6}$ Department of Medicine, Weill Medical College of Cornell University, New York, New York \\ ${ }^{7}$ Departments of Internal Medicine and/or Urology, Comprehensive Cancer Center, \\ University of Michigan, Ann Arbor, Michigan
}

\begin{abstract}
An unbiased cDNA expression phage library derived from bone-marrow endothelial cells was used to identify novel surface adhesion molecules that might participate in metastasis. Herein we report that reticulocalbin 1 (RCN1) is a cell surface-associated protein on both endothelial (EC) and prostate cancer (PCa) cell lines. RCN1 is an $\mathrm{H} / \mathrm{KDEL}$ protein with six EF-hand, calcium-binding motifs, found in the endoplasmic reticulum. Our data indicate that RCN1 also is expressed on the cell surface of several endothelial cell lines, including human dermal microvascular endothelial cells (HDMVECs), bone marrow endothelial cells (BMEC), and transformed human bone marrow endothelial cells (TrHBMEC). While RCN1 protein levels were highest in lysates from HDMVEC, this difference was not statistically significant compared BMEC and TrHBMEC. Given preferential adhesion of PCa to bone-marrow EC, these data suggest that RCN1 is unlikely to account for the preferential metastasis of PCa to bone. In addition, there was not a statistically significant difference in total RCN1 protein expression among the PCa cell lines. RCN1 also was expressed on the surface of several PCa cell lines, including those of the LNCaP human PCa progression model and the highly metastatic PC-3 cell line. Interestingly, RCN1 expression on the cell surface was upregulated by tumor necrosis factor alpha treatment of bone-marrow endothelial cells. Taken together, we show cell surface localization of RCN1 that has not been described previously for either PCa or BMEC and that the surface expression on BMEC is regulated by pro-inflammatory TNF- $\alpha$. J. Cell. Biochem. 104: 2298-2309, 2008. @ 2008 Wiley-Liss, Inc.
\end{abstract}

Key words: endothelial cells; prostate; LNCaP; progression; neoplasm; bone metastasis; phage display; reticulocalbin 1

Contract grant sponsor: Prostate Cancer SPORE; Contract grant number: P50 CA 69568; Contract grant sponsor: The University of Michigan Comprehensive Cancer Center; Contract grant number: CA 46592 (KP); Contract grant sponsor: NIH-K22 Career-Transition Award; Contract grant numbers: 5K22CA971117-3, CA096788; Contract grant sponsor: National Foundation for Cancer Research Center for Metastasis Research (CRC); Contract grant sponsor: University of Delaware; Contract grant sponsor: NIH/NCI; Contract grant numbers: PO1 CA098912, DAMD-17-00-1-0049, CA-105435, DK63919, CA-60948; (c) 2008 Wiley-Liss, Inc.
Contract grant sponsor: NIHNCRRINBRE; Contract grant number: RR016472-04; Contract grant sponsor: NIH Extramural Research Facilities Program; Contract grant numbers: C06RR14516, RO1-HL55267.

*Correspondence to: Dr. Carlton R. Cooper, PhD, Department of Biological Sciences, University of Delaware, 324 Wolf Hall, Newark, DE 19716. E-mail: crcooper@udel.edu Received 14 November 2007; Accepted 17 March 2008 DOI 10.1002/jcb.21785 
The goal of this study was to identify unique cell surface molecule(s) expressed on bonederived endothelial cells (BMEC) involved in prostate cancer (PCa) metastasis by phage display. Phage display is a powerful technique used to identify molecules involved in cell-to-cell and cell-to-extracellular matrix (ECM) interactions, both in vitro and in vivo [Koivunen et al., 1999]. By presenting fusion peptides on the surface of filamentous bacteriophage, libraries of peptides can be created and screened to determine their roles in cell adhesion [Smith and Scott, 1993; Koivunen et al., 1994]. Instead of the commonly used random peptide phage library, we used a phage cDNA expression library derived from an established bonemarrow endothelial cell line because it would allow for the identification of proteins expressed by these cells that bind to PCa cells. Through selective and subtractive biopannings, we identified reticulocalbin-1 (RCN1) as a potential BMEC surface molecule. Based on the literature, RCN1 is localized to the endoplasmic reticulum. In current study, we tested the hypothesis that RCN1 is expressed on the surface of endothelial and PCa cells and correlates with $\mathrm{PCa}$ progression. The expression of CAM on the surface of endothelial cells is regulated by cytokines, particularly proinflammatory cytokines previously shown to function in bone [Cooper et al., 2002, 2003]. Tumor necrosis factor-alpha (TNF- $\alpha$ ) upregulates the surface expression of several important CAMs, including vascular cellular adhesion molecule (VCAM), intracellular adhesion molecule (ICAM) and E-selectins. TNF- $\alpha$ biosynthesis is increased in patients with advanced PCa [Cooper et al., 2002]. Therefore, we examined the level of RCN1 expressed on the surface of bone-marrow endothelial cells treated with TNF- $\alpha$.

\section{MATERIALS AND METHODS}

\section{Reagents and Kits}

Reagents and kits used in this study were purchased from Invitrogen (Carlsbad, CA) unless otherwise noted. For mRNA- enrichment, cDNA synthesis, and phage display, kits were purchased from Novagen (Madison, WI).

\section{Cell Lines}

Immortalized human dermal microvascular endothelial cells (HDMVECs) were supplied by
Dr. Alvin Schmair University of Michigan (Ann Arbor, MI) and maintained in defined keratinocyte media with L-glutamine enhanced with a growth supplement, 20\% (v/v) fetal bovine serum (FBS) and 1\% (v/v) penicillin/ streptomycin (pen/strep). HBME-1 cells and WiDr colon cancer lines (American Type Culture Collection [ATCC], Manassas, VA) were maintained in Dulbecco's Modified Eagle Media (DMEM) with high glucose, GLUTAMAX ${ }^{\mathrm{TM}}$, $110 \mathrm{mg} / \mathrm{L}$ sodium pyruvate, pyridoxine- $\mathrm{HCl}$, $10 \%(\mathrm{v} / \mathrm{v})$ FBS, $1 \%$ (v/v) pen/strep, and $100 \mu \mathrm{l}$ of plasmocin, an anti-mycoplasma agent (Invitrogen) [Lehr and Pienta, 1998]. BMEC were a gift of Dr. Graca Almeida-Porada (University of Nevada School of Medicine, Reno, NV) [Almeida-Porada and Ascensao, 1996]. Cultures were maintained in Medium 199 with Earles's salts, L-glutamine, 2,200 $\mathrm{mg} / \mathrm{L}$ sodium bicarbonate, $25 \mathrm{mM}$ HEPES buffer, 10\% FBS, $1 \%$ pen/strep, endothelial cell growth supplement (BD Biosciences, Bedford, MA), and $7,500 \mathrm{u} / 500 \mathrm{ml}$ media of heparin (Sigma, St. Louis, MO). The TrHBMEC were maintained as previously described [Schweitzer et al., 1997] in DMEM media, with 5\% FBS and 10 mM HEPES buffer. Most importantly, at some time, the HBME cell line became contaminated with canine cells that were being grown concurrently in the laboratory. Because we could not ascertain with confidence when this occurred, all subsequent and reported experiments were performed with the BMEC and TrHBMEC cell lines that were clearly demonstrated to be of human origin by DNA sequence analysis of RCN1 RT-PCR products. LNCaP, C4-2, and C4-2B4 sublines from the LNCaP progression model of $\mathrm{PCa}$ [Thalmann et al., 2000] were maintained in $\mathrm{T}$ media with $5 \%(\mathrm{v} / \mathrm{v})$ FBS without antibiotics. The PC-3 (PCa cell line) and WiDr (colon cancer cell line) cell lines were obtained from ATCC. The PC-3 cell line was maintained in Ham's F-12 medium with $2 \mathrm{mM}$ L-glutamine, 1,500 mg sodium bicarbonate, $10 \%(\mathrm{v} / \mathrm{v})$ FBS and $1 \%(\mathrm{v} / \mathrm{v})$ pen/strep. All cell lines were maintained at $37^{\circ} \mathrm{C}$ in a $5 \% \mathrm{CO}_{2}$ incubator.

\section{RNA Isolation and cDNA Synthesis}

Briefly, total RNA was isolated from confluent cells by the Trizol method and quantitated using Smart Spec 3000 (Bio-Rad, Hercules, CA). For use in the T7 select phage display system, total RNA was enriched for mRNA 
transcripts using the Straight A's mRNA Isolation System. The mRNA transcripts were transcribed into cDNAs using the Novagen directional synthesis kit and oligo dT primers. For ratiometric RT-PCR and quantitative RT-PCR, cDNAs, $4 \mu \mathrm{g}$ of total RNA were used for the iScript ${ }^{\mathrm{TM}} \mathrm{cDNA}$ synthesis kit with oligo dT primers.

\section{Construction and Biopanning of a Bone-Marrow Derived Endothelial Phage Expression Library}

The protocols for phage display and biopanning are illustrated in Figure 1. Bone-marrow endothelial cell derived cDNAs were inserted by directional cloning into a T7Select10-3b vector. Phage display was performed as described in the manufacturer instructions with modifications. The bone-marrow endothelial phage library was biopanned on both WiDr cells, a non-metastatic colon cancer cell line, and on PC-3 cells, a PCa cell line derived from a bone metastasis. Subtractive biopanning on WiDr cell monolayers was performed to eliminate phage particles with non-specific binding activity. WiDr cells were grown to confluence in 96 -well plates. The media was removed and the monolayers were incubated for $20 \mathrm{~min}$ at $4^{\circ} \mathrm{C}$ in blocking buffer (phosphate-buffered saline (PBS) with $1 \%(\mathrm{w} / \mathrm{v})$ bovine serum albumin (BSA)). Approximately $10^{9}$ plaqueforming units of the BME phage expression library were added to the blocking solution and incubated over the WiDr monolayers for $1 \mathrm{~h}$ at $4{ }^{\circ} \mathrm{C}$. The monolayers were washed five times and the supernatants were pooled after each wash. The supernatant, which contained phage particles that were not able to bind to WiDr monolayers, was then applied to PC-3 cell monolayers. PC-3 cell monolayers were incubated in blocking buffer for $20 \mathrm{~min}$ at $4{ }^{\circ} \mathrm{C}$ and then the pooled supernatant from the WiDr monolayers was applied and allowed to incubate for $1 \mathrm{~h}$. After five washes with wash buffer (PBS with $0.1 \%(\mathrm{v} / \mathrm{v})$ Tween 20$)$, the attached phage were eluted with T7 elution buffer for $20 \mathrm{~min}$, then amplified by infecting $25 \mathrm{ml}$ of log phase BLT5403 bacterial cells. The resulting phage titer was determined by the plaque assays described in the T7 Select system manual and biopanning was repeated twice on PC-3 monolayers. Following the last biopanning, the attached phage were eluted and amplified in BLT5403 bacterial cells. Plaque assays were performed and random colonies were eluted in phage extraction buffer $(20 \mathrm{mM}$ Tris $-\mathrm{HCl}$, $\mathrm{pH}$ 8.0, $100 \mathrm{mM} \mathrm{NaCl}, 6 \mathrm{mM} \mathrm{MgSO}_{4}$ ) overnight

\section{Expression Phage Display}

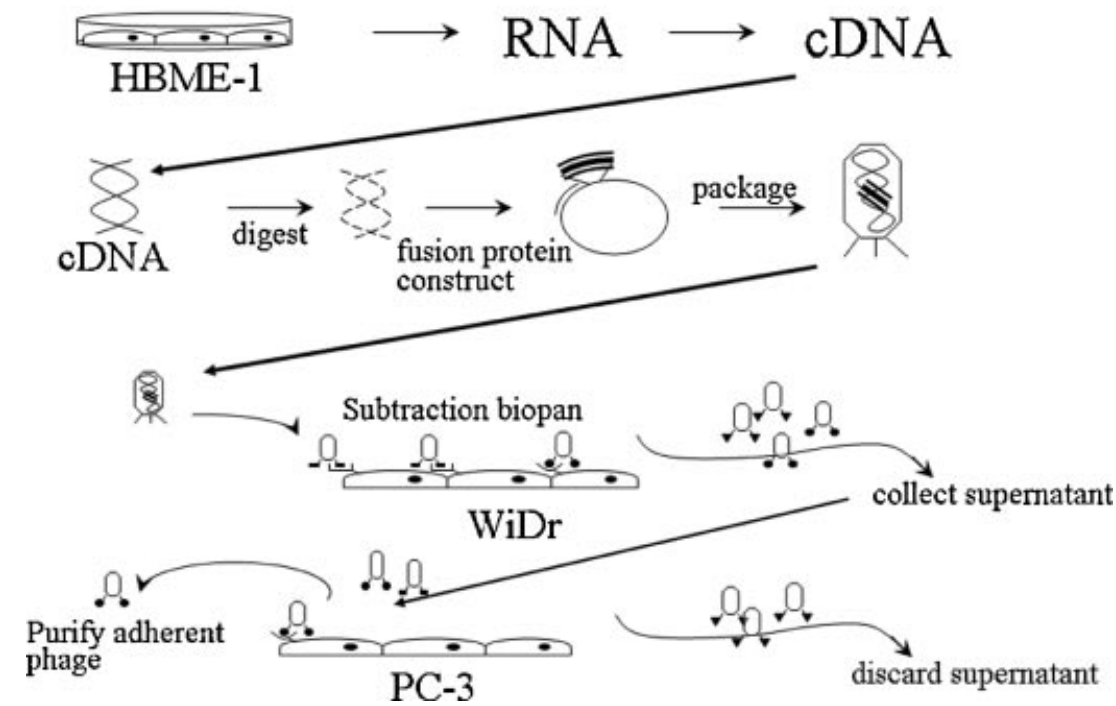

Fig. 1. Construction of the HBME Phage display library. Total RNA was isolated from HBME cells, reverse transcribed to CDNA, digested and packaged in a T7 Select10-3b phage vector. The HBME T7 phage expression vectors were grown in BLT5403 bacteria and a subtractive biopanning was performed on monolayers of WiDr cells. The non-adherent phage were collected and used for three rounds of conventional biopanning on monolayers of PC-3 cells. This step was enriched for phage expressing cDNA that preferentially bound to PC-3 cells. After several washes, phage attached to PC-3 cells were eluted, PCR amplified and sequenced as detailed in the materials and methods section. 
at $4^{\circ} \mathrm{C}$. Insert sizes were determined by PCR using T7 forward and reverse primers and nucleotide sequences were determined at the University of Michigan's DNA Sequencing Core Facility.

\section{RT-PCR and Sequence}

RT-PCR for RCN1 expression was performed using specific primers (forward 5'-GAGGACAACCAGAGCTTCCA- $3^{\prime}$ and reverse $5^{\prime}$-TCTGCCAGTTCAGTCCACAG- $3^{\prime}$ ) (MWG Biotech, Greensboro, NC) to generate a 1750 bp product. As a loading control, $\beta$-actin primers (forward $5^{\prime}$-GCTCG TCGTCGACAACCGGCTC- $3^{\prime}$ and reverse 5'-CAAACATGATCTGGGTCATCTTCTC-3') were used to generate a 353 bp product. Each RT-PCR reaction utilized $4 \mu \mathrm{g}$ of total RNA. RCN1 was amplified by PCR using $10 \%$ (corresponding to $0.4 \mu \mathrm{g}$ total RNA) of the cDNA reaction for each cell line using the SuperScript $^{\text {TM }}$ III First Strand RT-PCR kit using a Platinum Taq DNA polymerase and 30 cycles. PCR products were separated on a $0.8 \%$ TBE agarose gel and visualized with ethidium bromide. RT-PCR was performed on all endothelial cell (EC) lines, all of the cell lines of the LNCaP progression model, PC-3 cells and cDNAs from the following human organs: bone marrow, brain (cerebellum), kidney, and prostate (BD Biosciences, Bedford, MA).

Specificity of selected primers used for RTPCR amplification of RCN1 was confirmed by direct DNA sequencing. Four PCR reactions were performed utilizing the aforementioned protocol on each of the cell lines in the LNCaP progression model. The PCR-products were gel purified using low melt agarose (Bio-Rad) and QIAquick Gel Extraction kit (Qiagen, Valencia, $\mathrm{CA}$ ). Sequencing was performed in both directions using the ABI BigDye Terminator Cycle sequencing Ready Reaction kit v3.1 using a onefourth dilution of the terminator mix, and analyzed on an ABI3130XL Genetic Analyzer. Sequences generated during this study were assembled and analyzed using MacVector7.1.1 (Accelrys, San Diego, CA) and the BLAST program [Altschul et al., 1990] available on the National Center for Biotechnology Information (NCBI) web site. Secretome P 2.0 was used to predict the secretion of RCN1 http:// www.cbs.dtu.dk/services/secretomep/. A value greater than 0.5 strongly suggest that a protein is secreted [Bendtsen et al., 2004]. Also, the presence of transcription factor binding sites in the RCN1 promoter was determined using MatInspector/TRANSFAC from Baylor College of Medicine Search Launcher (http://searchlauncher.bcm.tmc.edu/seq-search/ gene-search.html.)

\section{Q-PCR}

To compare RCN1 transcript levels in the cell lines, quantitative real-time Q-PCR was performed. Approximately, $1 \times 10^{6}$ cells were trypsinized and rinsed in PBS. Cells then were lysed and total RNA was isolated by centrifugation through spin columns using the SV Total RNA Isolation System (Promega). cDNA was generated $(4 \mu \mathrm{g}$ total cellular RNA per reaction) by reverse transcription using Oligo (dT) 15 primer (Promega) and SuperScript III (500 units per reaction; Invitrogen) for first strand synthesis and Tfl DNA polymerase for second strand synthesis (500 units per reaction; Promega) as per the manufacturer's protocol. Typically, $20 \mathrm{pg}$ of each cDNA was used for each PCR reaction. Two-step Q-PCR was performed on cDNA generated using the MultiScribe reverse transcriptase from the TaqMan reverse transcription system and the SYBR Green PCR Master Mix (PE Biosystems). The primer pairs for RCN1 were generated from the published sequences (forward 5'-TGCAGACCTCAATGGTGACCT- $3^{\prime}$ and reverse $5^{\prime}$-AAGGCAGTGAACTCCTCCCG-3'). Reactions were performed in MicroAmp Optical 96-well Reaction Plate (PE Biosystems). Forty cycles of PCR were performed under standard conditions using an annealing temperature of $60^{\circ} \mathrm{C}$. The cycle threshold $(\mathrm{Ct})$ was determined by the cycle number at which exponential amplification began. The $\mathrm{Cts}$ were averaged from the values obtained from the triplicate repeats used to calculate delta $\mathrm{Ct}$ normalized to $\beta$-actin for endothelial cells and GAPDH in PCa cells using TrHBMEC (TR) as endothelial controls and $\mathrm{LNCaP}$ as controls for PCa cells. All reactions were carried out in triplicate.

\section{Western Blotting}

Proteins from cell lysates were obtained from confluent monolayers that had been washed 3 times with PBS before lysing in $20 \mathrm{mM}$ Tris (pH 8) containing $137 \mathrm{mM} \mathrm{NaCl}, 1 \%(\mathrm{v} / \mathrm{v}) \mathrm{NP} 40$, $10 \%(\mathrm{v} / \mathrm{v})$ glycerol, $1 \mathrm{mM} \mathrm{Na} \mathrm{VO}_{4}, 1.7 \mu \mathrm{g} / \mu \mathrm{l}$ aprotinin, $50 \mu \mathrm{l} / \mathrm{ml}$ leupeptin, and $1 \mathrm{mM} \mathrm{PMSF}$. Lysed cells were detached by scraping and the mixture was centrifuged at 13,200 rpm for 
$10 \mathrm{~min}$. Membrane proteins were obtained from confluent monolayers as described previously [Cooper et al., 2002]. The supernatant was collected and stored at $-80^{\circ} \mathrm{C}$. Protein concentrations were determined by a Bradford assay (Bio-Rad).

To determine protein expression of RCN1, $50 \mu \mathrm{g}$ of protein from the soluble fractions were resolved in $10 \%(\mathrm{w} / \mathrm{v})$ SDS-polyacrylamide gels on a Minigel apparatus (Bio-Rad) and transferred to a PVDF membrane (Immobilon ${ }^{\mathrm{TM}}-\mathrm{P}$, Millipore) using a semidry transfer (Bio-Rad). The membrane was blocked for $1 \mathrm{~h}$ with $3 \%(\mathrm{w} / \mathrm{v})$ BSA in TBS $(273.8 \mathrm{mM} \mathrm{NaCl}, 5.4 \mathrm{mM} \mathrm{KCl}$, and $38.1 \mathrm{mM}$ Tris) with $1 \%(\mathrm{v} / \mathrm{v})$ Tween 20 , the membrane was incubated with the primary antibody at the appropriate dilution, rabbit anti-reticulocalbin 1:3,000 (prepared commercially by Bethyl Laboratories, Montgomery TX and targets the EF-hand domain of the protein), rabbit anti-actin 1:5000 (Sigma), and rabbit anti- $\beta 1$-integrin 1:2,000 (Santa Cruz, Santa Cruz, CA), in $3 \%$ (w/v) BSA in TBS. Anti- $\beta 1-$ integrin was used as a loading control for membrane preparation. Anti-primary antibody exposure was carried out at $4^{\circ} \mathrm{C}$ for $18 \mathrm{hr}$ and membranes then washed 3 times with TBS-T (273.8 mM NaCl, 5.4 mM KCl, $38.1 \mathrm{mM}$ Tris, and $0.1 \%(\mathrm{v} / \mathrm{v})$ Tween 20$)$. For RCN1 detection, the membrane then was probed with goat antirabbit IgG HRP-linked 1:10,000 (Cell Signaling, Danvers, MA) for $1 \mathrm{~h}$ at room temperature and washed four times with TBS-T. For $\beta$-actin and $\beta 1$-integrin immunodetection, the membranes were probed with goat anti-rabbit IgG HRP-linked 1:20,000 (Cell Signaling), incubated for $1 \mathrm{hr}$ at room temperature, and washed four times with TBST. The immunoblots were visualized by chemiluminescence with LumiGlo $^{\circledR}$ (Cell Signaling). Membranes were treated with stripping buffer $(100 \mathrm{mM}$ $\beta$-mercaptoethanol, 2\% (w/v) SDS, $62.5 \mathrm{mM}$ Tris- $\mathrm{HCl}$ ) for $45 \mathrm{~min}$ at $70^{\circ} \mathrm{C}$, washed twice with TBST for $15 \mathrm{~min}$, then were reblocked and probed. All Westerns were done three times.

\section{Flow Cytometry}

Flow cytometric analyses were performed as described previously [Cooper et al., 2000, 2002]. Selected cell lines were seeded in T75 flasks and grown to confluence. For TNF- $\alpha$ treatment, BMEC and PCa cells were allowed to reach at least $80 \%$ confluency and then exposed to $400 \mathrm{U}$ of TNF- $\alpha$ for 4 and $24 \mathrm{~h}$. Cell monolayers were detached from plastic substrata with disadhesion medium $(0.5 \mathrm{mM}$ EDTA, $10 \mathrm{mM}$ glucose, $1 \times \mathrm{PBS}$ ) and approximately 2.5$5 \times 10^{5}$ cells were analyzed per run. After staining, cells were analyzed for fluorescence using a BD Bioscience FACSCalibur. The percentage of RCN-1 positive cells was determined using Cell Quest from Acquisition/ Analysis software (BD Bioscience). This experiment was done four times.

\section{Confocal Immunofluorescence Microscopy}

HBME-1, TrHBMEC and BMEC cells were grown on \#1.5 $18 \mathrm{~mm}$ coverslips to subconfluency prior to fixation with either $4 \%(\mathrm{v} / \mathrm{v})$ paraformaldehyde in $0.1 \mathrm{M}$ PBS $\mathrm{pH} 7.2$ or cold methanol $\left(-20^{\circ} \mathrm{C}\right)$ for $45 \mathrm{~min}$. For RCN1 endoplasmic reticulum (ER) labeling, paraformaldehyde-fixed cells were permeabilized with $0.1 \%(\mathrm{v} / \mathrm{v}$ ) Triton X-100 (Sigma, St. Louis, MO) for 10 min prior to immunolabeling. RCN1 and $\beta 1$-integrin detection at the plasma membranes were obtained after cold methanol or paraformaldehyde fixation, respectively, omitting Triton-X treatment. All samples were blocked in $3 \%(\mathrm{w} / \mathrm{v})$ BSA (Sigma) and 10\% (v/v) normal goat serum in PBS, pH 7.2 for $1 \mathrm{~h}$. Affinity-purified rabbit anti-RCN1 was used at a dilution of 1:500 in blocking buffer followed by Alexa Fluor 555 goat anti-rabbit IgG (A-21429, Invitrogen, Eugene, OR) at a dilution of 1:500 in blocking buffer with DRAQ-5 (Invitrogen) at 1:1,000 to visualize nuclei (in some experiments). Mouse monoclonal $\mathrm{IgG}_{1}$ anti- $\beta 1$-integrin (Santa Cruz) was used at a dilution of 1:100 followed by Alexa Fluor 488 goat anti-mouse $\operatorname{IgG}_{1}$ (Invitrogen) at a dilution of 1:500. All coverslips were mounted in Biomeda Gel/Mount (Electron Microscopy Sciences, PA) and sealed with nailpolish. Controls included the omission of primary antibody, secondary antibody, and/or peptide block (anti-RCN1 primary antibody incubated overnight in blocking peptide $-1 \mu \mathrm{l}$ antibody to $5 \mu \mathrm{l}$ peptide). Confocal images were acquired on a Zeiss Axiovert 200M equipped with a LSM 510 NLO laser-scanning microscope (Carl Zeiss, Inc., Germany) using a Zeiss 40X Plan-Neofluar objective lens (NA 1.3). Images of Alexa Fluor 488, Alexa Fluor 555 and/or DRAQ-5 staining were acquired using the $488 \mathrm{~nm}$ laser line of a $25 \mathrm{~mW}$ Argon laser (LASOS, Germany) and 543 and $633 \mathrm{~nm}$ Helium Neon lasers (LASOS) with the $500-550 \mathrm{~nm}$ band pass, $560-610 \mathrm{~nm}$ 
band pass and $650 \mathrm{~nm}$ long pass emission filters, respectively.

\section{Statistical Analysis}

Q-PCR results were statistically analyzed using the Student's $t$-test for unpaired values. $P$ values were determined by a two-sided calculation, and a $P$ value of less than 0.05 was considered statistically significant. Statistical differences are indicated by asterisks. Flow cytometry was analyzed using a paired Student $t$-test to determine statistical significance. A $P$ value of less than 0.05 was considered significant.

\section{RESULTS}

\section{RCN1 is Expressed in Established Endothelial Cell Lines}

In this study, a phage display expressed cDNA insert derived from a bone-marrow endothelial cell line was employed to identify proteins potentially involved in PCa cell adhesion. Several phage were identified containing cDNAs that adhered specifically to PC-3 cell monolayers. To enrich phage library for specific binding to PC-3, a subtractive biopanning on WiDr cells, which adhere poorly to HBME-1 cell monolayers, was done [2]. Using this approach, we identified a DNA sequence encoding a highly conserved calcium-binding domain corresponding to the EF-hand calcium-binding domain of human RCN1 (accession number BC010120) (data not shown).

To profile the expression of RCN1 in well characterized endothelial cell lines, Q-PCR analyses were performed using total RNA extracted from two independently derived bone-marrow endothelial cell lines, BMEC, and TrHBMEC, and one dermal microvascular endothelial cell line, HDMVEC (Fig. 2). Characterization of cell surface expression of VCAM and ICAM (data not shown) following TNF- $\alpha$ treatment, indicated that TrHBMEC and BMEC are more similar to primary human bone-marrow endothelium than are HBME-1; as such, they were used for all subsequent experiments [Almeida-Porada and Ascensao, 1996; Schweitzer et al., 1997; Lehr and Pienta, 1998; Cooper et al., 2002]. The data generated from Q-PCR showed that RCN1 mRNA was preferentially expressed in HDMVEC compared to bone-marrow endothelial cell lines (Fig. 2). The expression of RCN1 transcript

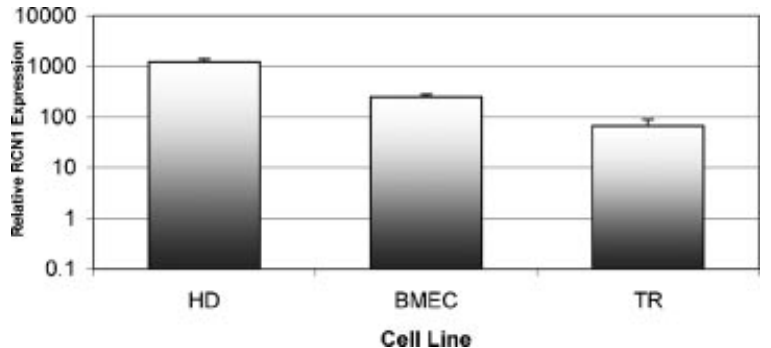

Fig. 2. The detection of RCN1 transcript expression in endothelial cell lines. $\beta$-Actin was used as a loading control. Q-PCR analysis of RCN1 expression in endothelial cell lines based on delta Cts (TR = TrHBMEC, BMEC, and HD= HDMVEC).

was 10 fold higher in the HDMVEC cell line compared to BMEC and TrHBMEC. BMEC expressed more RCN1 transcript compared to TrHBMEC.

To determine if RCN1 mRNA expression corresponded to RCN1 protein levels in these cell lines, Western blot analyses were performed using cell lysates and membrane preparations (Fig. 3). As with mRNA expression, RCN1 protein levels were highest in the HDMVEC cell lysate compared to bone-marrow endothelial cell lysates. Unlike RNA expression, BMEC expressed less RCN1 protein compared to TrHBMEC. Two bands were detected and were uniformly expressed in all endothelial cell lines. Interestingly, evaluation of membrane fractions derived from these endothelial cell lines demonstrated that HDMVEC expressed a high level of RCN1 compared to BMEC and TrHBMEC.

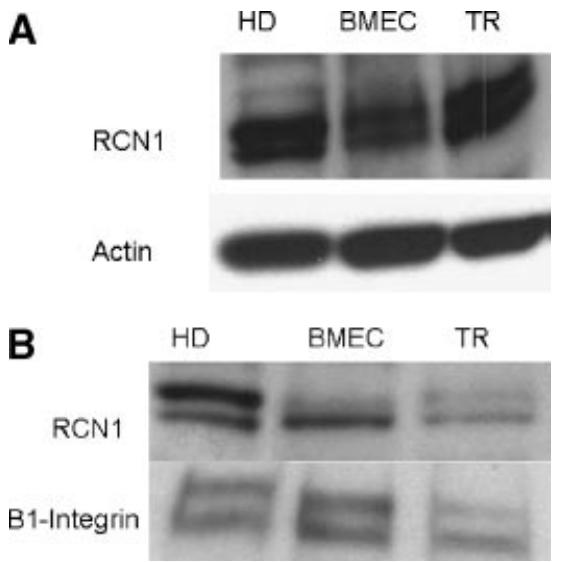

Fig. 3. Western analysis of RCN1 protein expression in endothelial cell lines. A: RCN1 in endothelial cell lysates (HDMVEC, TrHBMEC, and BMEC). B: RCN1 in endothelial cell membrane fractions. Fifty micrograms of proteins was resolved for both cell lysate and membrane fractions. 
RCN1 expression between the two bone-marrow EC were similar. Although two bands are detected and both are RCN1, the lower band was prevalent in the bone-marrow endothelial cell lines while the upper band was prevalent in HDMVEC. According to the manufacturer, both bands are RCN1 based on mass spectrometry. Actin was used as a loading control for cell lysates and $\beta 1$-integrin was used as a loading control for crude membrane preparations. These data demonstrated that RCN1 expression, although isolated by phage display from a bonemarrow endothelial cell line, is not unique to this cell type and may not contribute preferentially to PCa cells adhering to bone-marrow endothelium.

\section{RCN1 is Expressed in Established PCa Cell Lines}

RCN1 mRNA expression was assessed in established PCa cell lines by Q-PCR (Fig. 4). Here demonstrated a two-fold increase in RCN1 (Fig. 4A) associated with the progression from androgen sensitive ( $\mathrm{LNCaP})$ to androgen insensitive (C4-2). However, when comparing cells that are bone adapted, such as $\mathrm{C} 4-2$ versus C4-2B4 and PC-3, RCN1 mRNA expression was reduced by $60 \%$ and $50 \%$, respectively. Although the difference in RCN1 protein expression level was not statistically significant based on denisometric analysis (data not shown), there was a trend for increased RCN1 expression in osteoblastic C4-2B4 compared to osteolytic PC-3 cells (Fig. 4B). Finally, the level

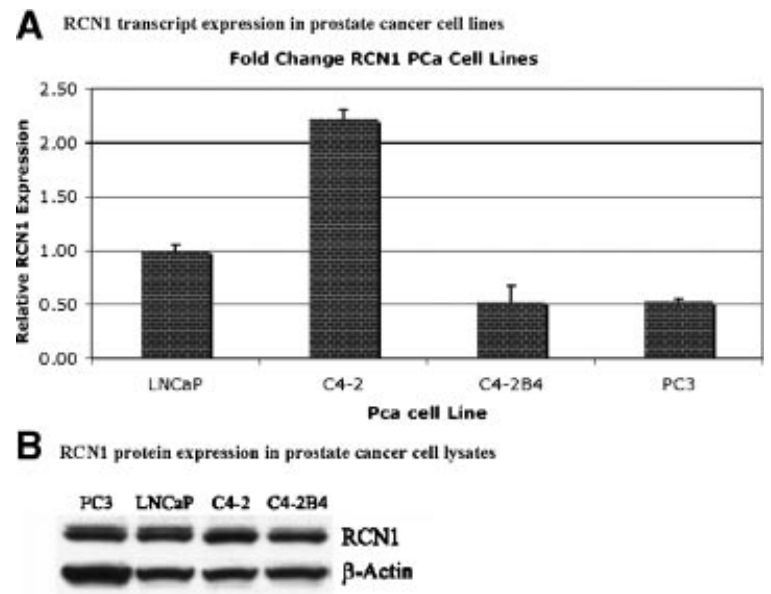

Fig. 4. Detection of RCN1 expression in PCa cell lines. A: Q-PCR analysis of RCN1 transcript expression in PCa cell lines based on average delta Cts. B: RCN1 protein expression in PCa cell lysates (PC-3, LNCaP, C4-2, and C4-2B4) as determined by Western analysis of $50 \mu \mathrm{g}$ of proteins resolved on a SDS gel. of protein expression did not correlate with the mRNA expression profile (Fig. 4B).

Pooled cDNAs derived from normal bonemarrow, brain, kidney and prostate tissues were examined for RCN1 expression using RTPCR (Fig. 5) to determine if RCN1 mRNA expression correlated with the metastatic pattern of $\mathrm{PCa}$ : bone $>$ brain $>$ kidney(rare). Only brain has noticeably higher RCN1 mRNA levels, while bone marrow actually appears to have a much lower level of RCN1 mRNA compared to the other tissues. These observations indicate that RCN1 transcript expression is common in normal tissues and the levels do not correlate with the metastatic pattern of PCa.

\section{RCN1 Is an ER Lumen Protein That Is Expressed at the Cell Surface}

$\mathrm{RCN} 1$ is a protein reported to reside in the ER lumen and has a classic ER retention signal (KDEL). Calreticulin also is found predominantly in the ER lumen, but also has been shown to be expressed in other sub-cellular compartments, including the plasma membrane where it participates in cell adhesion [Opas et al., 1996; Goicoechea et al., 2000; Johnson et al., 2001]. Based on published information regarding calreticulin, we evaluated our cell lines for the surface expression of RCN1. Despite the presence of an ER retention signal, we found RCN1 protein in membrane fractions of bone-marrow EC, indicating that RCN1 also could be associated with the plasma membrane. Flow cytometry on live EC and PCa cell lines confirmed that RCN1 was expressed on the surface of all EC lines tested (Table I). We found the highest surface expression on BMEC followed by HDMVEC and then TrHBMEC. In addition, we found significant surface expression of RCN1 on LNCaP and C4-2B4 cells, but lower levels in PC-3 cells.

\section{RCN1 expression in selected cDNA derived from normal tissue}

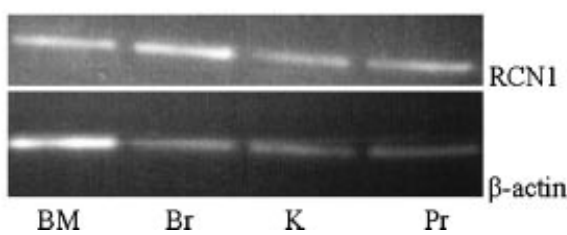

Fig. 5. The RCN1 transcript expression in normal human tissues ( $\mathrm{BM}=$ bone marrow, $\mathrm{Br}=$ brain (cerebellum), $\mathrm{K}=$ kidney, and $\mathrm{Pr}=$ prostate) as determined by conventional RT-PCR at 30 cycles. 
TABLE I. Relative Surface Expression of RCN1 on Endothelial and PCa Cell Lines Determined by FACS

\begin{tabular}{lc}
\hline Cell lines & $\begin{array}{c}\text { Average percentage of cells positive } \\
\text { for surface RCN1 expression }\end{array}$ \\
\hline TrHBMEC & $12 \pm 2.3$ \\
HDMVEC & $22 \pm 1.34$ \\
BMEC & $44 \pm 2.08$ \\
PC-3 & $22 \pm 5.2$ \\
LNCaP & $35 \pm 9.16$ \\
C4-2B4 & $38 \pm 2.8$ \\
\hline
\end{tabular}

Compilation of the FACS and Western data strongly suggest that RCN1 is expressed on the cell surface.

To validate the surface expression of RCN1, we used confocal microscopy to examine the subcellular distribution of RCN1 in bone-marrow ECs (Fig. 6). Figure 6A shows tubular staining indicating that RCN1 was prevalent in the ER of bone-marrow endothelial cell line HBME-1. We then demonstrated the presence of RCN1 on the plasma membrane of HBME-1 cells using methanol fixation (Fig. 6C). Treatment of Triton $\mathrm{X}-100$-treated and untreated HBME cells with peptide block prevented the RCN1 antibody from specifically binding to both ER RCN1 (Fig. 6B) and plasma membrane RCN1 (Fig. 6D), validating the specificity of RCN1 antibody. We also showed surface expression of RCN1 in TrHBMEC (Fig. 6G) and BMEC (Fig. 6H) cell lines to confirm it presence in human bone-marrow endothelia cells. To verify surface localization of RCN1, we examined cell surface expression of the $\beta 1$-integrin subunit that is known to be restricted to the cell surface [Cooper et al., 2000, 2002] in TrHBMEC (Fig. 6E) and BMEC (Fig. 6F) cells. Interestingly, RCN1 staining is enriched at the cell-tocell junctions in both BMEC and TrHBMEC, similar to $\beta 1$-integrin. These observations suggest the RCN1, is expressed both in the lumen of the ER and the surface of the plasma membrane in bone-marrow EC. This is the first report demonstrating surface expression of RCN1 on ECs, including those derived from bonemarrow, as well as PCa cells.

After demonstrating that RCN is expressed on the surface of bone-marrow endothelial cells, we evaluated the ability of TNF- $\alpha$ to upregulate the surface expression of RCN1 on BMEC endothelial cells (Fig. 7). Sequence analysis demonstrated that the promoter of RCN1 contains a binding site for transcription factors
(AP-1) regulated by pro-inflammatory cytokines. TNF- $\alpha$ was selected because its levels are upregulated in men with advanced $\mathrm{PCa}$ [Cooper et al., 2002; Nabors et al., 2003]. The data demonstrated that 4-24 h TNF- $\alpha$ treatment of BMEC produced a threefold increased in RCN1 surface expression, that approached statistical significant (the $\mathrm{p}$ value is 0.065 for the $4 \mathrm{~h}$ and 0.079 for the $24 \mathrm{~h}$ treatment). $\mathrm{PCa}$ cells with varied metastatic potential for bone were evaluated also for chronic ( $24 \mathrm{hrs}$ ) exposure to TNF- $\alpha$ (Table II). Although there was a trend for TNF- $\alpha$ to increase RCN1 surface expression on cells with high metastatic potential, PC-3 and C4-2B4, the increase was not statistically significance and not as dramatic as RCN1 surface expression observed on BMEC during acute $(4 \mathrm{~h})$ and chronic $(24 \mathrm{~h}) \mathrm{TNF}-\alpha$ stimulation. Thus, under pro-inflammatory conditions found in bone metastasis, RCN is highly expressed on bone-marrow endothelial cell surfaces (Table II).

\section{DISCUSSION}

PCa cell adhesion to microvascular endothelium is a necessary step in the cascade of events leading to metastasis [Lehr and Pienta, 1998; Cooper et al., 2000; Scott et al., 2001]. Although several integrins and lectins have been implicated in this process [Kierszenbaum et al., 2000; Scott et al., 2001], the specific cell adhesion molecules (CAMs) expressed on bone endothelium necessary for the adhesion of PCa cells remain unknown. Scott and colleagues [Scott et al., 2001] demonstrated that $\beta 1$-integrins were involved in PC-3 cell adhesion to bonemarrow endothelial cells (EC). In contrast, we have found that $\beta 1$-integrins expressed on receiving BME-1 cells were not involved in adhesion to bone-marrow endothelial cells [Cooper et al., 2000]. Kierszenbaum et al. [2000] showed that the galactosyl receptor, a cell surface C-type lectin, mediated PCa cell adhesion to BME-1 cells. These investigations indicate that several conventional cell surface molecules are involved in PCa-HBME interaction; however, other unique surface molecules also may be involved.

Bacteriophage display systems have been extensively used to isolate cell surface molecules [Koivunen et al., 1994; Koivunen et al., 1999; Romanov and Goligorsky, 1999]. Using phage display, we have identified a portion of RCN1 as a cell-surface interacting protein with 

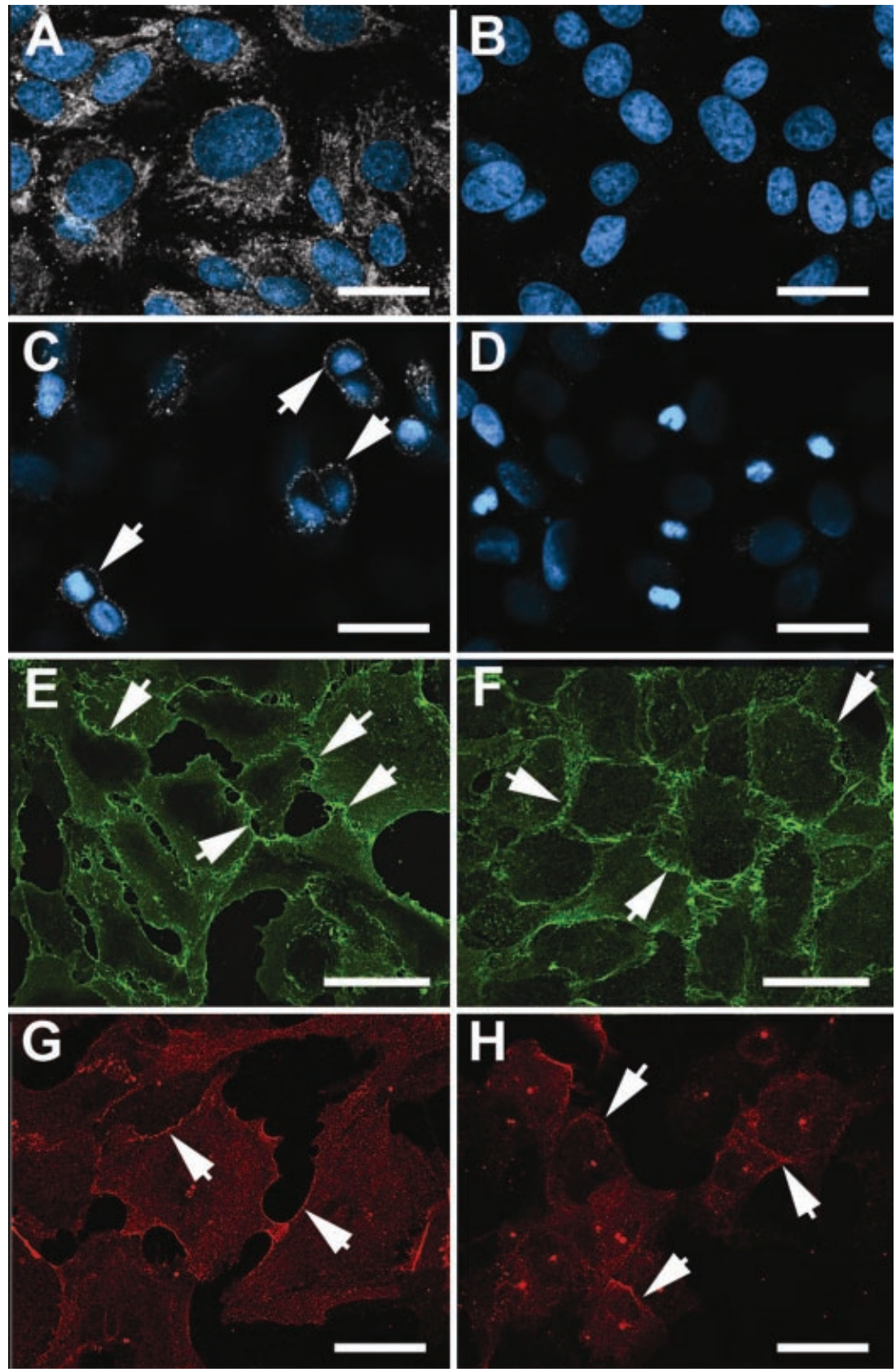

Fig. 6. Confocal microscopy examination of RCN1 in the ER

TrHBMEC. F: $\beta 1$-integrin subunit staining on BMEC. G: RCN1 and on the cell surface in three bone-marrow endothelial cell lines. A: RCN1 in the ER of HBME-1. B: RCN1 peptide block. C: RCN1 on the surface of non-HBME-1 (arrows). D: RCN1 peptide block. E: $\beta 1$-integrin subunit staining in

bone adapted PC-3 cells in vitro. Ozawa and Muramatsu [1993] first described human RCN1 as a protein with six $\mathrm{Ca}^{2+}$-binding domains [Tachikui et al., 1997] that is located in the lumen of the ER [Ozawa and Muramatsu, 1993]. The function of RCN1 is not known, but it has been speculated to play a role in $\mathrm{Ca}^{2+}$ staining on the cell surface of TrHBMEC. $\mathbf{H}$ : RCN1 staining on the cell surface of BMEC. The arrows indicate RCN1 surface location and cell-to-cell location. Scale Bars for $A-D=50 \mu \mathrm{m}$ and for $\mathrm{E}-\mathrm{H}=20 \mu \mathrm{m}$.

dependent cell adhesion [Nimmrich et al., 2000]. Strong expression of RCN1 correlates with a low expression of $\mathrm{Ca}^{2+}$-dependent cadherin, a metastatic predictor, in both breast cancer and colorectal cancer cells [Liu et al., 1997; Nimmrich et al., 2000]. A recent study reported that $\mathrm{RCN} 1$ is down-regulated in 
RCN-1 Surface Expression on BMECs

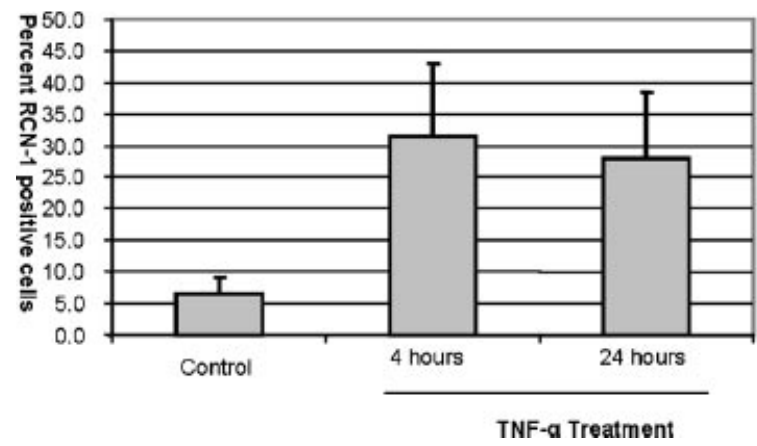

Fig. 7. FACS analysis of RCN1 surface expression on TNF- $\alpha$ treated BMECs. BMEC were treated for 4 and $24 \mathrm{~h}$ with $400 \mathrm{U}$ of TNF- $\alpha$. Control is untreated BMEC for $24 \mathrm{~h}$.

cisplatin-resistant non-small cell lung cancer tissue samples [Hirano et al., 2005]. Transfection of RCN1 into these cisplatin resistant cell lines restored sensitivity to cisplatin, suggesting that RCN1 enhances sensitivity of lung cancer cells to cisplatin. Although these studies provide insight into possible functions of RCN1, the exact function of RCN1 in normal and cancerous cells still is not known.

Herein we report for the first time that (1) RCN1 is expressed in EC lines, including bonemarrow ECs, (2) is expressed on the surfaces of EC cells and (3) is expressed on the surface of $\mathrm{PCa}$ cell lines. The surface expression was validated by flow cytometry and confocal microscopy. Interestingly, by FACS, we observed the BMEC expressed more surface RCN1 compared to TrHBMEC and HDMVEC. This is appears to contradict data generated from Western analysis of membrane preparation. One reason for this outcome is that the membrane preparation is crude and would contain Golgi and ER membranes, where as with FACS, only protein expression at the surface of the plasma membrane is being examined. Also, Western blot evaluation of membrane preparation reveal that the lower band is prevalent in the bonemarrow-derived EC compared to HDMVEC. This lower band may be the result of additional post-transational modification and locate predominately at the cell surfaces. While RCN1 has a KDEL sequence, which targets it to the lumen of the ER, several studies have reported the distribution of several KDEL containing proteins, including protein disulfide isomerase, a 78-kDa glucose-regulated protein (GRP78), and calreticulin, to the plasma membrane [Xiao et al., 1999; Johnson et al., 2001; Nabors et al., 2003]. Specifically, calreticulin, is expressed on the surface of bovine aortic ECs and interacts with thrombospondin to mediate focal adhesion disassembly [Goicoechea et al., 2000]. Like calreticulin, RCN1 at the plasma membrane may participate in cell adhesive events, as shown by its location at cell-to-cell junction in endothelial cells. However, because of the level of RCN1 protein expression in HDMVEC, it is unlikely that RCN1 is the candidate molecule responsible for preferential adhesion of $\mathrm{PCa}$ to bone endothelium.

Also, we are the first to demonstrate RCN1 expression in a PCa progression model system. RCN1 is over expressed in an invasive breast cancer cell line, MDA-MB-435, and in an invasive colorectal cancer cell line, SW480 [Liu et al., 1997; Nimmrich et al., 2000]. These observations imply that RCN1 expression correlated with cancer progression; however, these studies examined RNA expression and used a non-linear cell models for cancer progression. Using the lineage related $\mathrm{PCa}$ sublines of the human LNCaP PCa progression model system, we found very similar levels of RCN1 proteins among PCa cell lines even though there were differences in steady state mRNA levels. For example, mRNA expression was lower in more aggressive, bone adapted $\mathrm{PCa}$ cell lines (PC-3 and C4-2B4) than in those that were derived from the bone-marrow metastases compared to RCN1 mRNA expression in LNCaP and C4-2. However, the protein expression of RCN1 was approximately similar between $\mathrm{LNCaP}$, C4-2 and C4-2B4 cells. Using flow cytometry, we demonstrated that RCN1 was expressed on the cell surface in a subpopulation of PCa cells. Its

TABLE II. The Surface Expression of RCN1 on PCa Cells With Varying Metastatic Potential During Chronic (24 h) TNF- $\alpha$ Exposure*

\begin{tabular}{lccccccc}
\hline LNCaP & LNCaP-TNF $\alpha$ & C4-2 & C4-2TNF $\alpha$ & C4-2B4 & C4-2B4-TNF $\alpha$ & PC3 & PC3-TNF $\alpha$ \\
\hline $100 \pm 11$ & $114 \pm 8$ & $100 \pm 32$ & $110 \pm 24$ & $100 \pm 83$ & $156 \pm 83$ & $100 \pm 70$ & $182 \pm 64$ \\
\hline
\end{tabular}

*Data are presented at fold change relative to untreated control for each cell line. 
expression was the highest in $\mathrm{LNCaP}$ and C4-2B4 and lowest in PC3, suggesting that its surface expression does not correlate with $\mathrm{PCa}$ progression to the bone.

Inflammation is a common complication in cancer patients and upregulates surface expression of several CAMs on proximal microvascular endothelial [Fayth et al., 2007]. In the current study, we showed that the surface expression of RCN1 on bone-marrow EC and bone-marrowderived PCa cells was increased in the presence of TNF- $\alpha$, a pro-inflammatory cytokine that is often over-expressed in men with advanced metastatic PCa [Cooper et al., 2002]. This observation suggests that the expression of RCN1 on the endothelial cell and metastatic $\mathrm{PCa}$ surfaces is increased under the proinflammatory condition often found in bone metastasis and may contribute to metastatic progression in this tissue. An antibody to RCN1 failed to block the adhesion of PC-3 to BMEC, suggesting that RCN1 may not be a mediator of PCa-BMEC adhesion (data not shown). However, it should be noted that the antibody used was against the EF-hand region of the protein and has not been reported to have blocking function.

In conclusion, while mRNA levels encoding RCN1 are not useful markers for PCa progression or for identification of surface receptive endothelial cell populations, cell surface levels of RCN1 may serve as useful indicators of proadhesive endothelial cells. Additional studies are underway to determine the functional significance of cell surface RCN1 and its presentation at the cell surface by inflammatory mediators associated with $\mathrm{PCa}$ metastasis to bone.

\section{ACKNOWLEDGMENTS}

We would like to thank Drs. Carolyn Schanen and Melinda Duncan for additional assistance with sequence analysis. We are grateful to Dr. Randall Duncan and Dr. Danny Welch for helpful discussions. Finally, we acknowledge the many helpful discussions of the Prostate Cancer Working Group at the University of Delaware. This work was supported by the following sources: The University of Michigan's Comprehensive Cancer Center SPORE grant P50 CA 69568 and Comprehensive Cancer Grant CA 46592 (KP); a NIH-K22 CareerTransition Award, 5K22CA971117-3 (CRC) and start-up funds from the University of Delaware (CRC, RAS) and NIH/NCI PO1 CA098912, subproject 2 (MCF). Additional partial support was provided by DAMD-17-001-0049, CA-105435, DK63919 (RAS), CA-60948 (JAM, HC), NIHNCRRINBRE RR016472-04 (KC, CRC), NIH-K22 Career-Transition AwardCA096788 and NIH Extramural Research Facilities Program grant C06RR14516 (CD), and RO1-HL55267 (BW).

\section{REFERENCES}

Almeida-Porada G, Ascensao JL. 1996. Isolation, characterization, and biologic features of bone marrow endothelial cells. J Lab Clin Med 128:399-407.

Altschul SF, Gish W, Miller W, Myers EW, Lipman DJ. 1990. Basic local alignment search tool. J Mol Biol 215: 403-410.

Bendtsen JD, Jensen LJ, Blom N, Von Heijne G, Brunak S. 2004. Feature-based prediction of non-classical and leaderless protein secretion. Protein Eng Des Sel 17: 349-356.

Cooper CR, McLean L, Mucci NR, Poncza P, Pienta KJ. 2000. Prostate cancer cell adhesion to quiescent endothelial cells is not mediated by beta-1 integrin subunit. Anticancer Research 20:4159-4162.

Cooper CR, Bhatia JK, Muenchen HJ, McLean L, Hayasaka S, Taylor J, Poncza PJ, Pienta KJ. 2002. The regulation of prostate cancer cell adhesion to human bone marrow endothelial cell monolayers by androgen dihydrotestosterone and cytokines. Clin Exp Metastasis 19:25-33.

Cooper CR, Chay CH, Gendernalik JD, Lee HL, Bhatia J, Taichman RS, McCauley LK, Keller ET, Pienta KJ. 2003. Stromal factors involved in prostate carcinoma metastasis to bone. Cancer 97:739-747.

Goicoechea S, Orr AW, Pallero MA, Eggleton P, MurphyUllrich JE. 2000. Thrombospondin mediates focal adhesion disassembly through interactions with cell surface calreticulin. J Biol Chem 275:36358-36368.

Hirano T, Kato H, Maeda M, Gong Y, Shou Y, Nakamura M, Maeda J, Yashima K, Kato Y, Akimoto S, Ohira T, Tsuboi M, Ikeda N. 2005. Identification of postoperative adjuvant chemotherapy responders in non-small cell lung cancer by novel biomarker. Int J Cancer 117:460468

Johnson S, Michalak M, Opas M, Eggleton P. 2001. The ins and outs of calreticulin: From the ER lumen to the extracellular space. Trends Cell Biol 11:122-129.

Kierszenbaum AL, Rivkin E, Chang PL, Tres LL, Olsson CA. 2000. Galactosyl receptor, a cell surface C-type lectin of normal and tumoral prostate epithelial cells with binding affinity to endothelial cells. Prostate 43:175183.

Koivunen E, Wang B, Dickinson CD, Ruoslahti E. 1994. Peptides in cell adhesion research. Methods Enzymol 245:346-369.

Koivunen E, Arap W, Rajotte D, Lahdenranta J, Pasqualini R. 1999. Identification of receptor ligands with phage display peptide libraries. J Nucl Med 40:883888 
Lehr JE, Pienta KJ. 1998. Preferential adhesion of prostate cancer cells to a human bone marrow endothelial cell line. J Natl Cancer Inst 90:118-123.

Liu Z, Brattain MG, Appert H. 1997. Differential display of reticulocalbin in the highly invasive cell line, MDA-MB-435, versus the poorly invasive cell line, MCF-7. Biochem Biophys Res Commun 231:283289.

Nabors LB, Suswam E, Huang Y, Yang X, Johnson MJ, King PH. 2003. Tumor necrosis factor alpha induces angiogenic factor up-regulation in malignant glioma cells: A role for RNA stabilization and HuR. Cancer Res 63:4181-4187.

Nimmrich I, Erdmann S, Melchers U, Finke U, Hentsch S, Moyer MP, Hoffmann I, Muller O. 2000. Seven genes that are differentially transcribed in colorectal tumor cell lines. Cancer Lett 160:37-43.

Opas M, Szewczenko-Pawlikowski M, Jass GK, Mesaeli N, Michalak M. 1996. Calreticulin modulates cell adhesiveness via regulation of vinculin expression. J Cell Biol 135: 1913-1923.

Ozawa M, Muramatsu T. 1993. Reticulocalbin, a novel endoplasmic reticulum resident calcium-binding protein with multiple EF-hand motifs and a carboxyl-terminal HDEL sequence. J Biol Chem 268:699-705.

Romanov VI, Goligorsky MS. 1999. RGD-recognizing integrins mediate interactions of human prostate carcinoma cells with endothilial cells in vitro. Prostate 39:108-118.

Schweitzer KM, Vicart P, Delouis C, Paulin D, Drager AM, Langenhuijsen MM, Weksler BB. 1997. Characterization of a newly established human bone marrow endothelial cell line: Distinct adhesive properties for hematopoietic progenitors compared with human umbilical vein endothelial cells. Lab Invest 76:25-36.

Scott L, Clarke N, George N, Shanks J, Testa N, Lang S. 2001. Interactions of human prostatic epithelial cells with bone marrow endothelium: Binding and invasion. Br J Cancer 84:1417-1423.

Smith GP, Scott JK. 1993. Libraries of peptides and proteins displayed on filamentous phage. Methods Enzymol 217:228-257.

Tachikui H, Navet AF, Ozawa M. 1997. Identification of the calcium-binding domains in reticulocalbin, an endoplasmic reticulum resident calcium-binding protein with multiple EF-hand motifs. J Biochem 121:145-149.

Thalmann GN, Sikes RA, Wu TT, Degeorges A, Chang SM, Ozen M, Pathak S, Chung LW. 2000. LNCaP progression model of human prostate cancer: Androgen-independence and osseous metastasis. Prostate 44(2):91-103.

Xiao G, Chung TF, Fine RE, Johnson RJ. 1999. Calreticulin is transported to the surface of NG 108-15 cells where it forms surface patches and is partially degraded in an acidic compartment. J Neurosci Res 58:652-662. 